\title{
A phylogenetic study of Drosophila splicing assembly chaperone RNP-4F associated U4-/U6-snRNA secondary structure
}

\author{
Jack C. Vaughn ${ }^{*}$, Sushmita Ghosh, Jing Chen \\ Department of Biology, Cell Molecular and Structural Biology Program, Miami University, Oxford, USA; \\ *Corresponding Author: vaughnjc@MiamiOH.edu
}

Received 15 August 2013; revised 23 September 2013; accepted 1 October 2013

Copyright (C) 2013 Jack C. Vaughn et al. This is an open access article distributed under the Creative Commons Attribution License, which permits unrestricted use, distribution, and reproduction in any medium, provided the original work is properly cited.

\section{ABSTRACT}

The rnp-4f gene in Drosophila melanogaster encodes nuclear protein RNP-4F. This encoded protein is represented by homologs in other eukaryotic species, where it has been shown to function as an intron splicing assembly factor. Here, RNP-4F is believed to initially bind to a recognition sequence on U6-SnRNA, serving as a chaperone to facilitate its association with U4-SnRNA by intermolecular hydrogen bonding. RNA conformations are a key factor in spliceosome function, so that elucidation of changing secondary structures for interacting snRNAs is a subject of considerable interest and importance. Among the five snRNAs which participate in removal of spliceosomal introns, there is a growing consensus that U6-snRNA is the most structurally dynamic and may constitute the catalytic core. Previous studies by others have generated potential secondary structures for free U4- and U6-snRNAs, including the Y-shaped U4-/U6-snRNA model. These models were based on study of RNAs from relatively few species, and the popular Y-shaped model remains to be systematically re-examined with reference to the many new sequences generated by recent genomic sequencing projects. We have utilized a comparative phylogenetic approach on 60 diverse eukaryotic species, which resulted in a revised and improved U4-/U6-snRNA secondary structure. This general model is supported by observation of abundant compensatory base mutations in every stem, and incorporates more of the nucleotides into base-paired associations than in previous models, thus being more energetically stable. We have extensively sampled the eukaryotic phylogenetic tree to its deepest roots, but did not find genes potentially encoding either U4- or U6-snRNA in the Giardia and Trichomonas data-bases. Our results support the hypothesis that nuclear introns in these most deeply rooted eukaryotes may represent evolutionary intermediates, sharing characteristics of both group II and spliceosomal introns. An unexpected result of this study was discovery of a potential competitive binding site for Drosophila splicing assembly factor RNP-4F to a 5'-UTR regulatory region within its own premRNA, which may play a role in negative feedback control.

Keywords: RNP-4F; snRNA Secondary Structure; U4-/U6-snRNA Phylogeny; Spliceosome Evolution

\section{INTRODUCTION}

Drosophila melanogaster, which is a cosmopolitan holometabolous insect found in all warm environments, has been an important model organism for genetic, molecular, cellular and physiological studies for over a century. Its small size (usually 2 - $4 \mathrm{~mm}$ ), short life cycle (10 - 14 days at $25^{\circ} \mathrm{C}$ ), high reproductive rate (an adult female can lay 400 - 500 eggs in 10 days), completely sequenced and largely annotated genome, well-developed techniques, and evolutionarily-conserved molecular pathways all contribute to making Drosophila a research paradigm. It has been predicted that about $75 \%$ of human disease genes have clear homologs in D. melanogaster $[1,2]$, an observation leading to the extensive use of Drosophila which has led to advances in the improvement of human health.

The long-term objective of our research is to understand evolutionarily-conserved cellular, developmental, molecular and genetic mechanisms behind regulation of 
genes which encode intron splicing assembly factor proteins, a topic about which relatively little is known. The system which we are currently using to address these questions is the Drosophila $r n p-4 f$ gene, which encodes splicing assembly factor RNP-4F, and we are concentrating on mechanisms of posttranscriptional level regulation [3-11]. This protein is believed to play a direct role during spliceosome assembly by acting as a chaperone to unwind U6-snRNA and thus facilitate its association with U4-snRNA via intermolecular hydrogen bonding [12-16]. In the course of our work, we became interested in secondary structure interactions within the Drosophila U4-/U6-snRNA duplex.

The major or U2-type molecular pathway for removal of spliceosomal introns has been extensively studied [reviewed in 17, 18], and shown to require direct participation of five trans-acting small nuclear uracil-rich RNAs (snRNAs) termed U1, U2, U4, U5 and U6. These RNAs are each associated with specific sets of proteins to yield the corresponding biologically active snRNPs, which progressively interact with pre-mRNAs and with each other during the ensuing spliceosomal assembly. In addition to these snRNAs, about 70 different snRNP proteins and more than 100 non-snRNP proteins have been shown to be spliceosomal components [reviewed in 19]. For example, the essential Saccharomyces cerevisiae premRNA splicing protein Prp24, represented in Drosophila by its ortholog RNP-4F and in human by p110 [13,14] facilitates U4- and U6-snRNA pairing during spliceosomal assembly [16].

A succession of snRNA conformational changes accompanies steps in the splicing pathway, which are essential in generation and function of the catalytic structure. Elucidation of the changing secondary structures of the interacting snRNA molecules is therefore a subject of considerable interest and importance. The comparative phylogenetic approach [20,21] generates models in which existence of potential biologically significant stem-loops can be established by observation of compensatory base mutations in diverse species, and has proven to be a powerful technique. The original Y-shaped U4-/U6-snRNA duplex secondary structure model [12] was based on this methodology by comparing yeast, fruit-fly, plant and human sequences. Subsequent studies have shown that RNAs from various species can also be folded in accordance with this model [22-26]. However, no attempt has ever been made to systematically re-examine the original model itself, utilizing the relative abundance of new sequences now available for analysis.

\section{MATERIALS AND METHODS}

\subsection{Selection of U4- and U6-snRNA Sequences}

We began by utilizing the original Small RNA Data- base [27] as a source for sequences published early. We then carried out GenBank searches, followed by BLAST searches (http://www.ncbi.nlm.nih.gov/BLAST) in which bait sequences were derived from the major phylogenetic levels. Finally, the number of sequences available for study was further increased from early published work not submitted to GenBank. The BLAST search was more successful in finding U6-snRNAs, owing to their extremely high sequence conservation. We did not use every sequence found, excluding for example those from eleven other Drosophila species [28] and also different species of Saccharomyces, since their inclusion would add little additional understanding due to having virtually identical sequences within a genus. This exercise (Table 1) yielded 42 U4- and 56 U6-snRNAs, of which 38 were both available in a given species and deemed optimal for our study. In total, sequences from some 60 different species were included in our study.

\subsection{Alignment of U4- and U6-snRNA Sequences}

All sequences selected for this study were individually aligned with reference to the corresponding Drosophila genes using the ClustalW program (http://align.genome.jp), and the resulting alignment was further refined by eye. Finally, the alignment was adjusted using the emerging secondary structure results, to assure that homologous nucleotides would be compared for evidences of compensatory base mutations. The final alignments (not shown) included as few deletions (gaps) and insertions as possible, while generating the maximum number of matching residues.

\subsection{Strategy for U4-/U6-snRNA Duplex Secondary Structure Determination}

We elected to start completely from the beginning in deriving our secondary structure model, in contrast to merely modifying existing models, to optimize the chances of identifying structural components not previously recognized. We began by utilizing version 3.6 of the Mfold program (http://mfold.rna.albany.edu) [29] for the two genes individually from Drosophila melanogaster (fruit-fly), Homo sapiens (human), Arabidopsis thaliana (plant), Kluyveromyces lactis (yeast) and Trypanosoma brucei (flagellate). GenBank accession numbers are given in Table 1. These structures contained a variety of potential stem-loops, and were combined to include only stem-loops held in common. The resulting U4- and U6-snRNA structures were then combined to accommodate base-pairing between the two molecules in the two closely adjacent U6 locations previously determined by photochemical cross-linking in mammalian snRNAs [30] and by subsequent observation of compensatory base 
Table l. U4 and U6 RNA sequences utilized in this study.

\begin{tabular}{|c|c|c|}
\hline \multirow[t]{2}{*}{$\underline{\text { Organism }}$} & \multicolumn{2}{|c|}{ GenBank Accession Number or Reference } \\
\hline & U6-snRNA & U4-snRNA \\
\hline \multicolumn{3}{|l|}{$\underline{\text { Animalia, Vertebrate }}$} \\
\hline Homo sapiens (human) & X07425 & X59361 \\
\hline Pan troglodytes (chimpanzee) & AC146131 & NW_001223167 \\
\hline Macaca mulatta (monkey) & NW_001218112 & NW_001096649 \\
\hline Mus musculus (mouse) & X06980 & AC159539 \\
\hline Rattus norvegicus (rat) & AC120800 & K00477 \\
\hline Canis familiaris (dog) & AC188530 & NW_876282 \\
\hline Bos taurus (cattle) & NW_001492849 & NW_001493540 \\
\hline Sus scrofa (pig) & CR956385 & ----- \\
\hline Equus caballus (horse) & NW_001799704 & NW_001799734 \\
\hline Monodelphis domestica (opossum) & NW_001581906 & NW_001584232 \\
\hline $\begin{array}{l}\text { Ornithorhynchus anatinus } \\
\text { (duck-billed platypus) }\end{array}$ & NW_001794177 & NW_001765942 \\
\hline Gallus gallus (chicken) & NW_001471627 & M14136 \\
\hline Xenopus tropicalis (frog) & M31687 & ---- \\
\hline Danio rerio (zebrafish) & CU466287 & NW_001514552 \\
\hline \multicolumn{3}{|l|}{ Animalia, Invertebrate } \\
\hline Drosophila melanogaster (fruit-fly) & X06669 & D00043 \\
\hline Aedes aegypti (mosquito) & AAGE02013372 & ----- \\
\hline Anopheles gambiae (mosquito) & NZ_AAAB02008807 & ----- \\
\hline Culex pipiens (mosquito) & AAWU01008690 & AAWU01009244 \\
\hline Apis mellifera (honey bee) & NW_001253045 & ----- \\
\hline Nasonia vitripennis (jewel wasp) & NW_001815737 & AAZX01001234 \\
\hline Bombyx mori (silkworm moth) & AADK01011346 & DQ861919 \\
\hline Tribolium castaneum (flour beetle) & AC154132 & NW_001092869 \\
\hline $\begin{array}{l}\text { Tachypleus tridentatus } \\
\text { (horseshoe crab) }\end{array}$ & \multicolumn{2}{|c|}{ (horseshoe crab) } \\
\hline Ascaris lumbricoides (nematode) & L22252 & L22250 \\
\hline Caenorhabditis elegans (nematode) & X07829 & X07828 \\
\hline Schistosoma mansoni (trematode) & L25920 & ---- \\
\hline Taenia solium (tapeworm) & AF529186 & ----- \\
\hline Lytechinus variegatus (sea urchin) & ----- & U37266 \\
\hline \multicolumn{2}{|l|}{ (sea urchin) } & NW_001323459 \\
\hline \multicolumn{3}{|l|}{ Fungi, Ascomycota } \\
\hline $\begin{array}{l}\text { Saccharomyces cerevisiae } \\
\text { (budding yeast) }\end{array}$ & X12565 & Siliciano et al. (1987) \\
\hline $\begin{array}{c}\text { Schizosaccharomyces pombe } \\
\text { (fission yeast) }\end{array}$ & X14196 & X15491 \\
\hline Kluyveromyces lactis & NC_006042 & Guthrie \& Patterson (1988) \\
\hline Candida albicans & EU144231 & EU144229 \\
\hline Vanderwaltozyma polyspora & NZ_AAZN01000268 & ---- \\
\hline
\end{tabular}




\begin{tabular}{|c|c|c|}
\hline Ashbya gossypii & NC_005788 & ----- \\
\hline \multicolumn{3}{|l|}{ Fungi, Basidiomycota } \\
\hline Erythrobasidium hasegawianum & Tani \& Ohshima (1991) & D63682 \\
\hline Puccinia graminis & AAWC01000866 & ----- \\
\hline Coprinopsis cinerea & AACS01000244 & ----- \\
\hline Phanerochaete chrysosporium & AADS01000210 & ----- \\
\hline \multicolumn{3}{|l|}{ Amoebozoa, Mycetozoa } \\
\hline \multicolumn{3}{|l|}{ (slime mold) } \\
\hline \multicolumn{3}{|l|}{ (slime mold) } \\
\hline \multicolumn{3}{|l|}{ Amoebozoa, Conosa } \\
\hline Entamoeba histolytica & U43841 & BK006131 \\
\hline \multicolumn{3}{|l|}{ Viridiplantae, Eudicot } \\
\hline Arabidopsis thaliana (thale cress) & X52527 & X67145 \\
\hline Vicia faba (broad bean) & Solymosy \& Pollak (1993) & Solymosy \& Pollak (1993) \\
\hline Pisum sativum (pea) & Solymosy \& Pollak (1993) & X15933 \\
\hline Solanum lycopersicum (tomato) & X51447 & ----- \\
\hline Solanum tuberosum (potato) & S83742 & ----- \\
\hline Populus trichocarpa (Poplar) & NC_008469 & NC_008470 \\
\hline \multicolumn{3}{|l|}{ Viridiplantae, Monocot } \\
\hline Oryza sativa (rice) & NC_008405 & DQ649301 \\
\hline Triticum aestivum (wheat) & X63066 & ----- \\
\hline Zea mays (maize) & ---- & Solymosy \& Pollak (1993) \\
\hline \multicolumn{3}{|l|}{ Viridiplantae, Algae } \\
\hline $\begin{array}{c}\text { Chlamydomonas reinhardtii } \\
\text { Alveolata, Cilliophora }\end{array}$ & X71486 & X71485 \\
\hline Tetrahymena thermophila & Orum et al. (1991) & Orum et al. (1991) \\
\hline \multicolumn{3}{|l|}{ Alveolata, Apicomplexa } \\
\hline Plasmodium falciparum & EF419774 & EF140769 \\
\hline \multicolumn{3}{|l|}{ Euglenozoa } \\
\hline Trypanosoma brucei (flagellate) & X57046 & Solymosy \& Pollak (1993) \\
\hline Crithidia fasciculata (flagellate) & X78550 & AF326336 \\
\hline Leishmania tarentolae (flagellate) & ---- & X97621 \\
\hline Leishmania mexicaca (flagellate) & X82228 & ---- \\
\hline Leptomonas seymouri (flagellate) & X78552 & AJ245951 \\
\hline Phytomonas sp. (flagellate) & X82229 & ----- \\
\hline
\end{tabular}

mutations [12], which resulted in further simplification of potential stem-loops in the predicted duplex RNA structure. Compensatory base changes were then entered onto the Drosophila duplex structure in comparison with the five species originally used to begin the study (above), using the alignment to assure that homologous nucleotides were being compared. We adopted the criterion [20] that existence of a helix is considered proven if there are at least two base-pair replacements. Stems as short as two base-pairs are acceptable if compensatory base changes can be demonstrated (Carl Woese, personal communication). Finally, the provisional model was compared to every species utilized in the study (Table 1), to determine the extent to which the resulting structure was universal. When an otherwise proven stem-loop was found to be absent from any taxonomic level, the timing 
of that loss was charted with reference to the eukaryotic phylogenetic tree [31].

\section{RESULTS AND DISCUSSION}

\subsection{An Improved General Secondary Structure Model for U4-IU6-snRNA}

The derived U4-/U6-snRNA duplex secondary structure model is shown in Figure 1, and structures from representative species at different taxonomic levels in Figures 2(a)-(h). A relatively large proportion of all nucleotides are base-paired in our U4-/U6-snRNA model. For example, in Drosophila 58\% are base-paired in U4 and 63\% in U6, whereas in the Y-shaped model the corresponding numbers are $58 \%$ and $33 \%$. Four stem-loops (I-IV) are found to be present in the U4 structure for most species, so that our model both confirms and extends the secondary structure for free U4-snRNA previously proposed [32] using the phylogenetic approach with far fewer species. The existence of stem-loop IV in free U4-snRNA, proposed by the same authors, is also confirmed for all species studied by us. The overall conformation of the structure shown in our model is very similar in every species examined, with the exception of stem-loop III in U4-snRNA which is further discussed in Section 3.2. Each stem in our model has been proven by

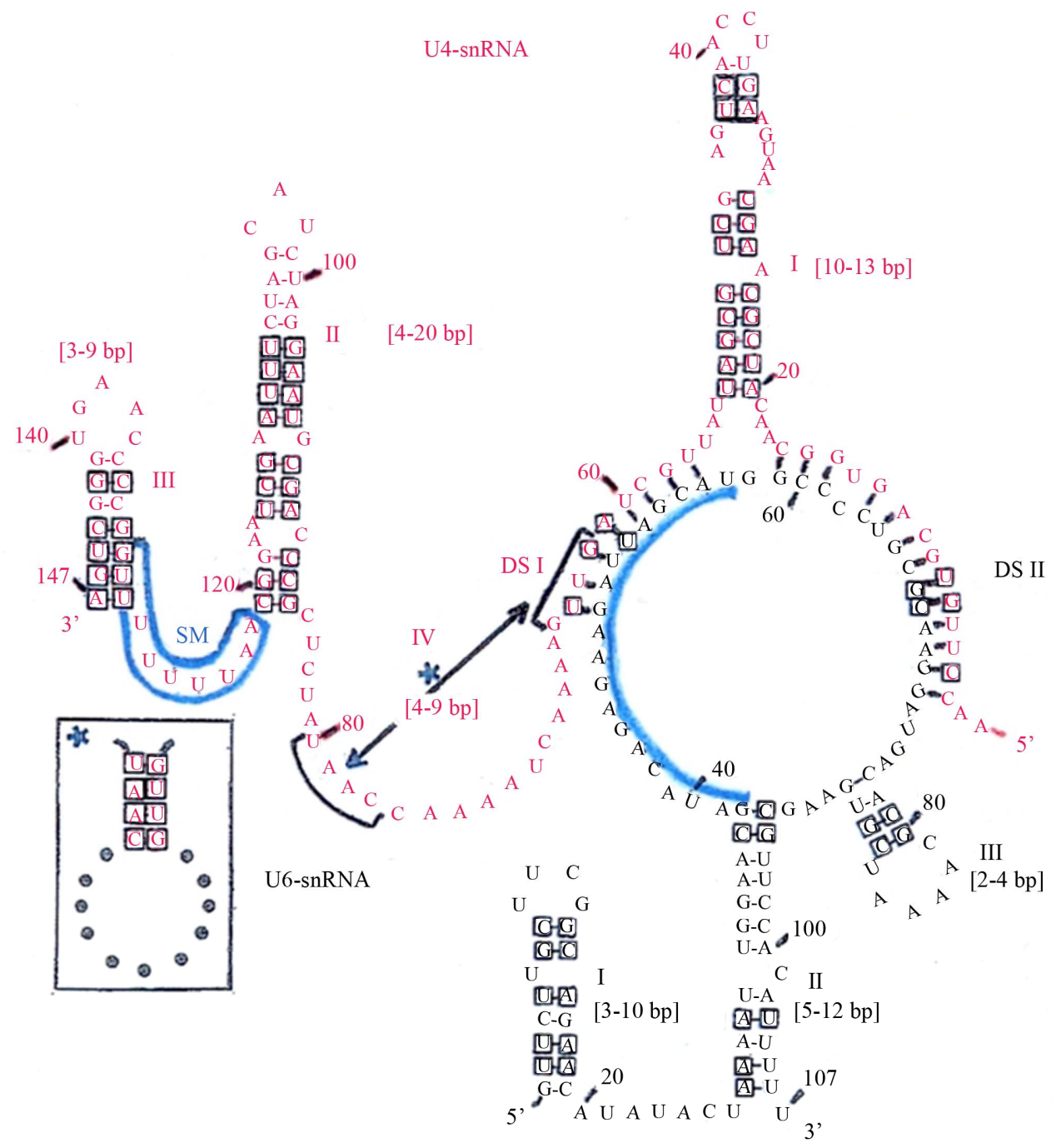

Figure 1. General secondary structure model for Drosophila U4-/U6-snRNA duplex. The two RNAs interact by base-pairing within regions designated DS I and DS II. Compensatory base changes which prove the structure illustrated are boxed and were identified in the alignment with reference to the structures derived for $H$. sapiens, A. thaliana, K. lactis and T. brucei. The range of stem lengths found between different species in our study is shown beside each stem. Stem-loop IV in free U4-snRNA (large box) is disrupted upon binding to U6-snRNA. The putative SM-binding site (SM) is indicated. An RNA recognition motif (RRM) in chaperone RNP-4F/Prp24/p110 binds primarily to a tract within free U6-snRNA nucleotides \#38-57 (13), which is indicated by a heavy vertical overlay. 
observation of numerous compensatory base mutations. Species within the flagellate group Euglenozoa were found to have the shortest overall U4- and U6-snRNA lengths (compare D. melanogaster in Figure 1 with $T$. brucei in Figure 2(h)). Despite the close similarity in conformation among species, nearly all stem lengths are however quite variable (Figure 1). The most consistent stem length is in U4 stem I, which ranges from 10 - 13 base pairs and is always interrupted by a structurally
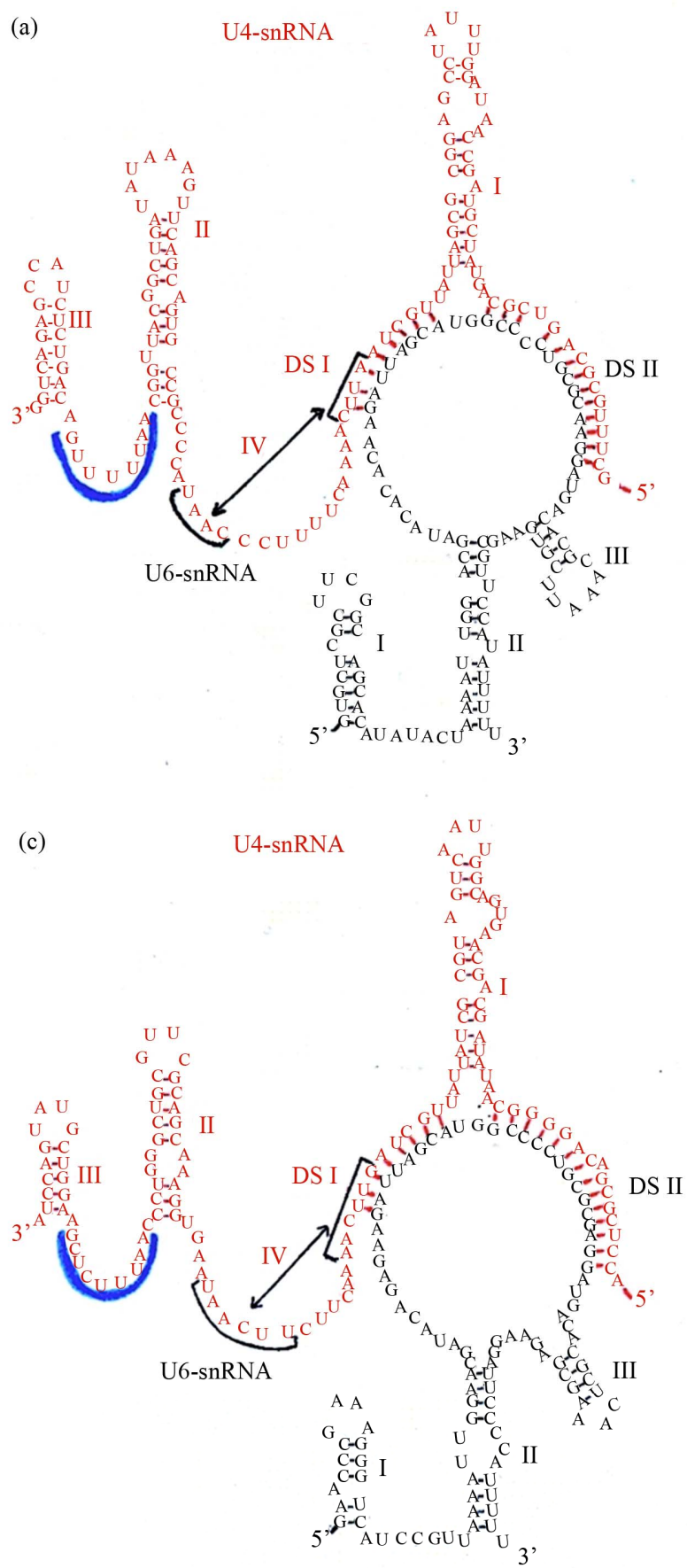

conserved bulge loop. A conspicuous highly conserved sequence tract in $\mathrm{U} 4$ is the putative SM-binding site, located near the 3'-end between stem-loops II and III, which matches the consensus sequence AU [4-6] G. Our study confirms the universality of the two major intermolecular base-paired zones of contact between the two RNA molecules (DS I and DS II) as originally proposed [12], with many examples of compensatory base mutations.
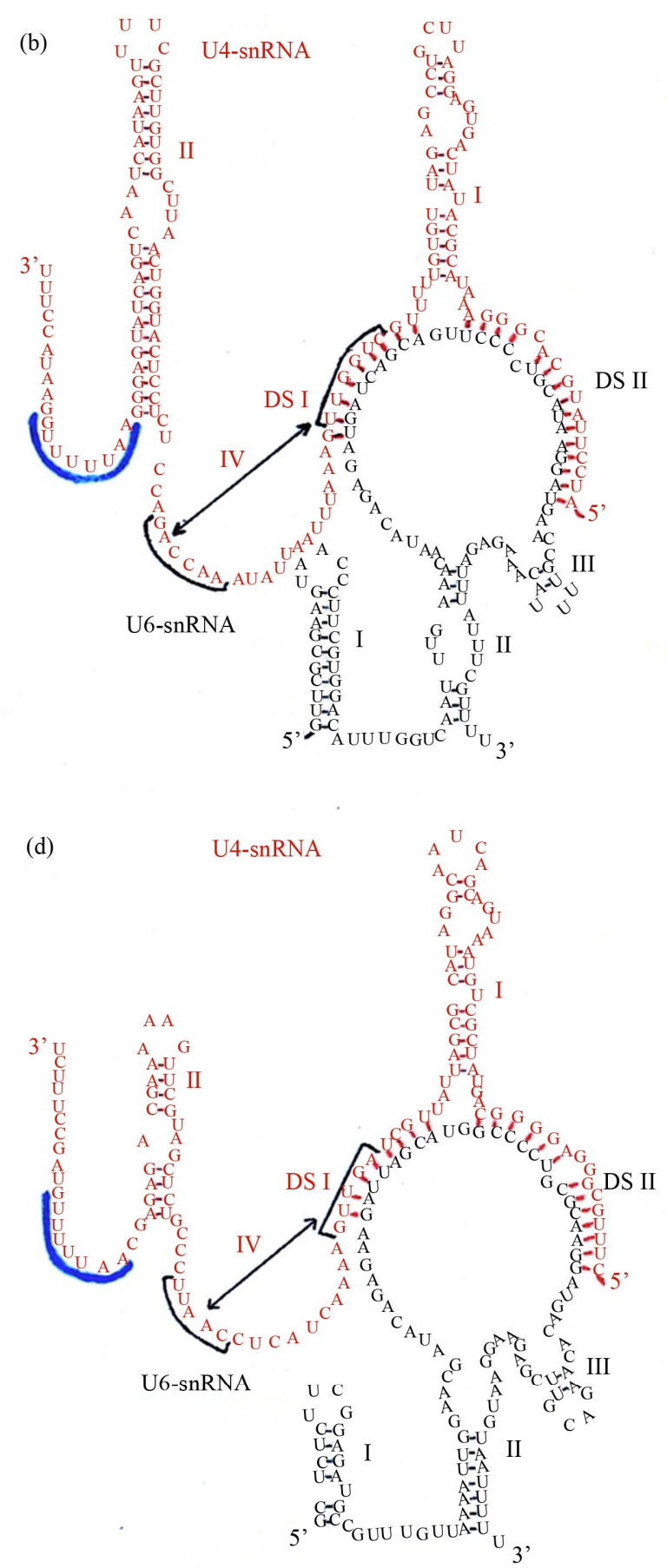

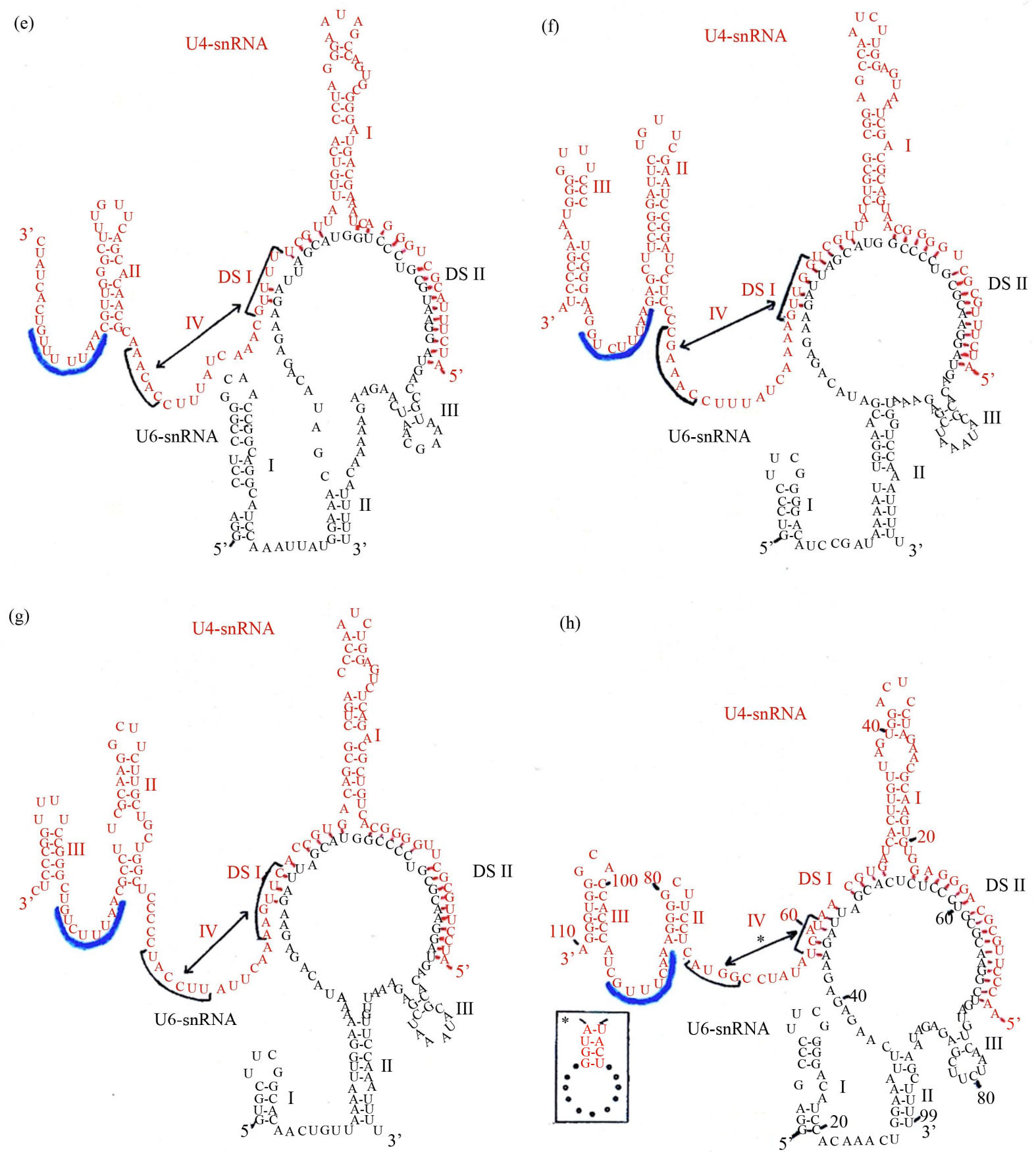

Figure 2. Representative U4-/U6-snRNA secondary structures from phylogenetically diverse species, folded according to our general model. (a) H. sapiens; (b) S. cerevisiae; (c) T. thermophila; (d) P. falciparum; (e) D. discoideum; (f) A. thaliana; (g) C. reinhardtii; (h) T. brucei. Labeling is as in Figure 1.

The U6-snRNA nucleotide sequence is relatively highly conserved, in comparison with that for U4. Three stem-loops are also present in the U6 structure in our duplex model, which is in contrast to the Y-shaped model in which only stem-loop I is shown. In our model the 3 '-end of U6-snRNA is incorporated into the structure to form stem-loop II in every species examined, albeit in some cases with a central bulge loop or absence of base-pairing at the top of the stem. U6 stem-loop II is proven by observation of compensatory base mutations, and is not shown in other models. A second U6 structural feature in our model which is not shown in other models is a short stem-loop III. This stem-loop is only two base-pairs long in many species, but is proven by obser- 
vation of compensatory base mutations. We did however fail to observe this stem-loop in the fungus $C$. albicans and in E. histolytica, showing that it is not universal.

\subsection{The General Secondary Structure Model is Not Universal and Multiple Independent U4-snRNA Stem-Loop III Losses Have Occurred During Evolution}

Representative structures for a diverse selection of evolutionarily distant species show that the general model is not universal. The most striking example is in the absence of U4-snRNA stem-loop III (Figures 2(b), (d), (e)), otherwise proven by observation of numerous compensatory base changes. The absence of this stemloop has previously been noted in secondary structures for various species of yeast and slime molds [12,25,32, 33]. It has been suggested that the absence of this stem-loop is correlated with phylogenetic depth, implying that this structural feature was not present in the earliest eukaryotes and is newly evolved [32]. We tested this hypothesis by superimposing the presence/ absence of this stem-loop onto the eukaryotic phylogenetic tree [31]. The results show that this stem-loop is present in all species among the deeply-rooted flagellate Euglenozoa examined, but that three clearly independent secondary losses have occurred during evolution (Figure 3). The most recent is within Fungi, where all Ascomycete species studied have lost the stem-loop, which is however present in the Basidiomycete E. hasegawianum. An earlier independent loss occurred among the Amoebozoa, where the Mycetozoa slime mold species examined have lost the stem-loop but the amoeboid Conosa E. histolytica has not. The earliest loss is in the Alveolata, where this feature is absent in the Apicomplexa P. falciparum but not in the Cilliophora T. thermophila.

\subsection{The General Secondary Structure Model Compared to the Classical Y-Shaped Model}

It is informative to compare the secondary structures of free U4- and U6-snRNAs with that of the duplex which is formed upon their association during spliceosome assembly, in consideration of the most parsimonious solution for their association (Figure 4). An excellent free U4-snRNA secondary structure model has previously been proposed based on the phylogenetic approach [32], utilizing a taxonomic diversity of species extending only as deep as the slime mold Physarum. This structure has been experimentally supported by the results of enzymatic digestion studies in rat U4-snRNA [34]. The model contains four stem-loops, of which three are incorporated directly into both our model and the
Y-shaped model. Stem-loop IV is disrupted in favor of intermolecular base-pairing to form DS I, upon association with U6-snRNA. Our results confirm and extend the previously proposed free U4-snRNA model, showing that the structure has been retained to its origin within the flagellate group Euglenozoa (Figure 3). There are no differences in this part of our model in comparison to the Y-shaped model.

Previously proposed free U6-snRNA models for human [35] and yeast $S$. cerevisiae [36] show somewhat differing structures, which are both supported by the results of chemical and enzymatic probing in these species [37]. In the simplest free U6-snRNA secondary structure model, as exemplified in Drosophila (Figure 1) and human, a short stem-loop is present at the 5'-end and the entire 3'-terminus is folded into one long interrupted stem-loop (Figure 4(a)). In human the chaperone p110, an ortholog of Drosophila RNP-4F, has been shown to bind primarily to free U6-snRNA nucleotides \#38-57 [13], promoting unwinding of the long stem-loop and base-pairing to two closely adjacent tracts on U4-snRNA, which we have designated as DS I and DS II, followed by chaperone release.

Our model and the Y-shaped model differ primarily in how they show the U6-snRNA structure within the RNA duplex. In the latter model, only the 5'-end stem-loop is retained (Figure 4(c)), and no base-pairing occurs elsewhere except within regions DS I and DS II, so that the 3 '-end is unpaired. In our model, the base of old free U6-snRNA is retained in stem-loop II, which brings the 3 '-end into a duplex structure (Figure 4(b)). One set of observations in support of this structure is seen in the compensatory mutations present in this stem (Figure 1). The results of previously reported chemical and enzymatic probing of the U4-/U6-snRNA duplex further support the model which we have proposed and not the Y-shaped model. In human, chemical reagent modifications were not observed within nucleotides \#27-38 or \#94-106, which comprise the helix in stem-loop II in our model but which are shown in long unpaired 5'- and 3'-tracts in the Y-shaped model. These observations are indicative of a double-stranded structure here, and this interpretation is confirmed by the observation of RNase $\mathrm{V}_{1}$ cleavage 3' to positions 33 and also 35 in the human U4-/U6-snRNA duplex [37]. This is an enzyme which cleaves specifically double-stranded RNA regions. These results have also been reported by these authors upon probing the base of free U6-snRNA stem-loop II. It has been proposed that a potential third base-paired region of contact may exist between U4- and U6-snRNA [24]. In this view, the top of our U6-snRNA stem-loop II is base-paired with a complement located within the long single-stranded U4 connective between DS I and U4 stem-loop II. We are skeptical of this proposed third zone 


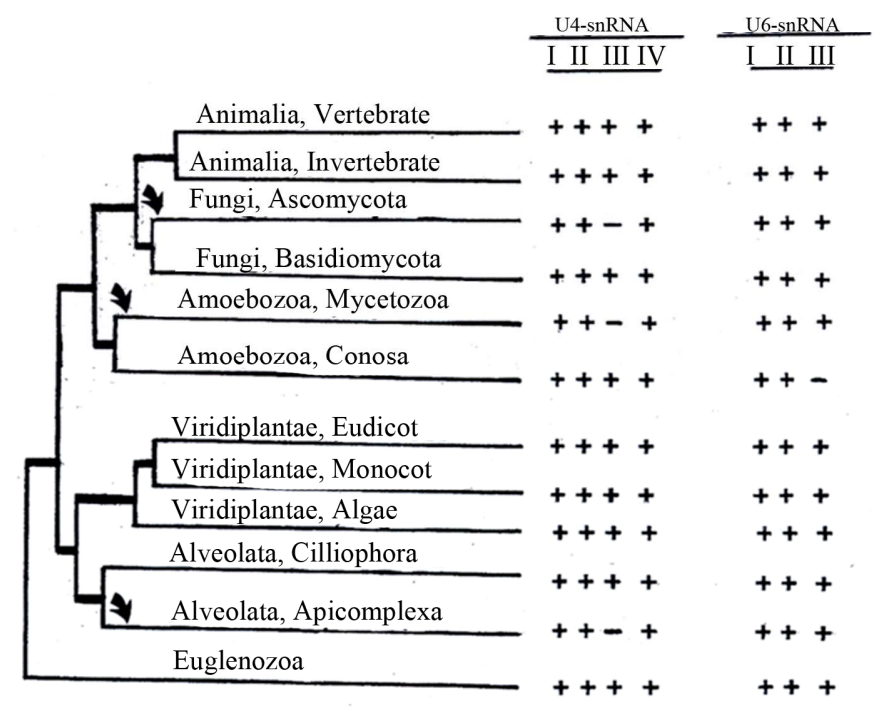

Figure 3. Eukaryotic phylogenetic tree (31), showing taxonomic distribution of species included in our study and stem-loops observed. U4-snRNA stem-loop III has been independently lost at least three times (arrows) during evolution of these RNAs.
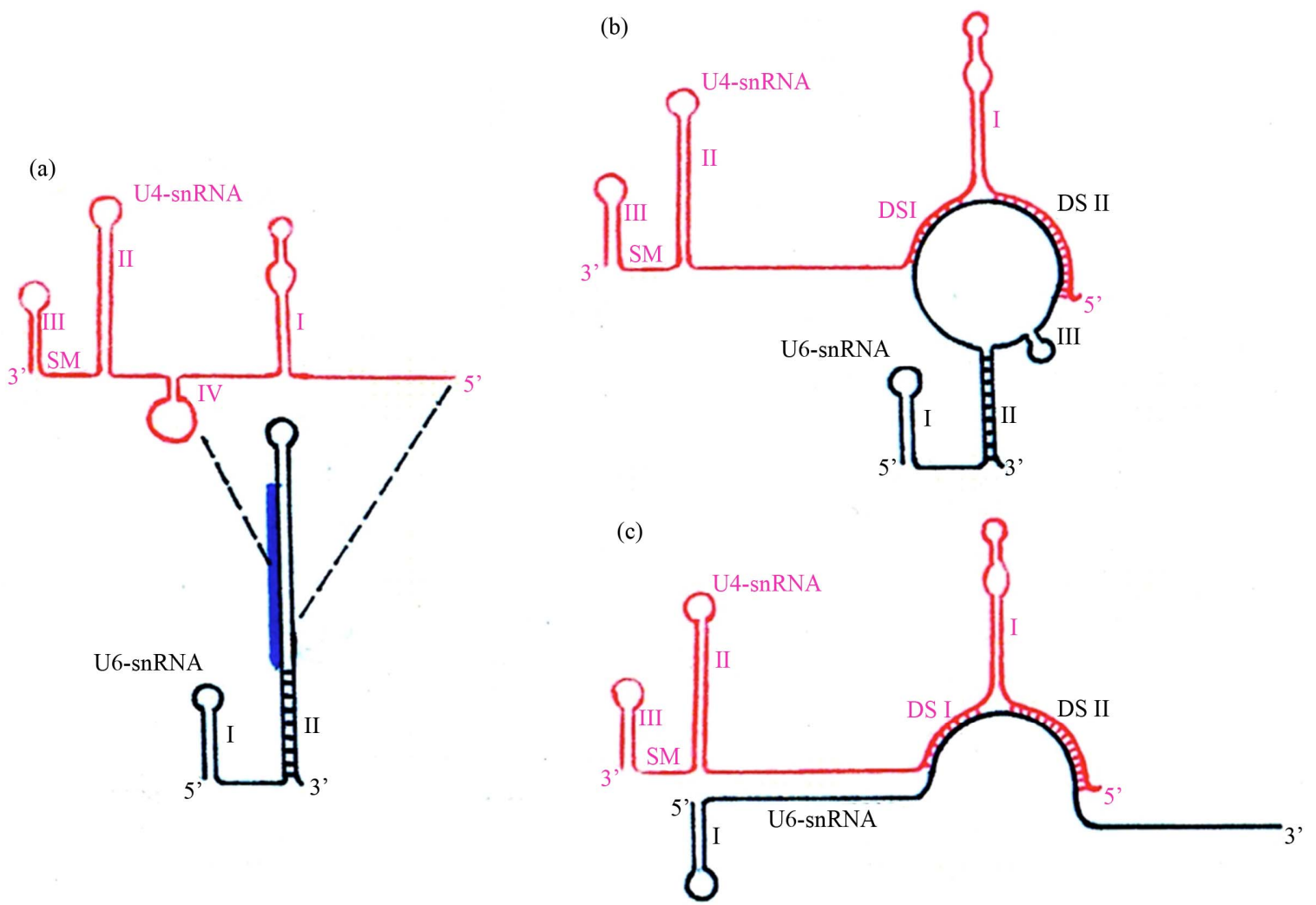

Figure 4. Comparison between our general U4-/U6-snRNA secondary structure and the Y-shaped model. (a) Structures of free U4- (32) and U6-snRNA (35) prior to their interaction. The primary position for binding of RRM in chaperone RNP-4F/Prp24/p110 to free U6 stem-loop II (13) is indicated by heavy vertical overlay, and was determined experimentally. The unwinding of U6 stem-loop II due to chaperone activity permits base-pairing between the two RNAs (region bounded by the broken lines). The base of stem-loop II (cross-bars) remains associated in the resulting duplex structure in our model. (b) Our general secondary structure model. (c) The Y-shaped model (12), shown inverted to facilitate comparisons. 
of RNA/RNA interaction, since different nucleotides in the alignment must be utilized to create this structure. For example, in $S$. cerevisiae and $K$. lactis the U4 region of contact is very different from that for other species.

Within free U6-snRNA, nucleotides comprising stemloop III are contained within the long stem-loop (Figure 4(a)). The existence of stem-loop III in the duplex structure is proven by observation of compensatory base mutations, but the stem length is reduced to only two basepairs in many species. Chemical and enzymatic probing of the human U4-/U6-snRNA duplex [37] did not provide any further clarification for existence of this stemloop, since most of this region was contained in the site of the primer utilized. Cryo-electron microscopy of isolated U4-/U6-snRNA has been reported to show two major structural domains linked by a thin connective [38], in good agreement with our general secondary structure model.

\subsection{Phylogenetic Depth of the Genes Encoding U4- and U6-snRNAs}

The secondary structure of the U4-/U6-snRNA duplex in our model is found to be identical, with the exception of multiple independent losses of U4 stem-loop III discussed above, down to and including the deeply-rooted flagellate group Euglenozoa. However, extensive BLAST searches against both the Giardia [39] and Trichomonas [40] genome sequences failed to detect any U4- or U6snRNA orthologs, using the corresponding T. brucei sequences as bait. The diplomonads and parabasalids are generally considered to be descendants of the earliest extant eukaryotes [31], leading us to consider the implications of this observation.

Success in BLAST searches is dependent on the degree of nucleotide conservation between bait and prey sequences, in addition to the completeness and accuracy of the genomic sequence database itself. The nucleotide sequences of genes encoding U6-snRNAs are among the most highly conserved of any eukaryotic genes. For example, the human and Drosophila U6-snRNA sequences are $94 \%$ identical. The U6-snRNA sequence within and immediately flanking the region of base-pairing with U4-snRNA is exceptionally well conserved. For example, comparison between Drosophila and flagellate T. brucei U6 nucleotides \#40-75 shows 86\% identity. This degree of conservation is far greater than that observed for U4, making identification of its most ancient orthologs more difficult. It was therefore surprising that no U6-snRNA genes turned up during BLAST searches against both the diplomonad and parabasalid genomes.

The Giardia and Trichomonas genome annotations are well along, and we therefore asked if ANY of the U-series snRNA gene sequences have been annotated in these species. Surprisingly, NONE of these genes have been found despite an $\sim 7 \mathrm{X}$ coverage during sequencing. In addition, none of the genes encoding proteins which are part of the U4- and U6-snRNPs in other eukaryotes have been found (Steven Sullivan, personal communication). Annotation of the Giardia genome has also failed to detect any genes encoding U4- or U6-snRNA (Hilary Morrison, personal communication). What are the implications of these observations? The spliceosome is widely viewed as having evolved from self-splicing group II introns like those in organellar protein-encoding genes as well as in many bacteria [reviewed in 41,42], which do not utilize the U-series of snRNAs. Interestingly, it has been proposed that Giardia and Trichomonas nuclear introns may represent evolutionary intermediates, showing characteristics of both group II and spliceosomal introns [43]. If so, then our study suggests that genes encoding U4- and U6-snRNAs, and the resultant duplex RNA which forms between them with a virtually identical secondary structure among all eukaryotes, may have evolved within the flagellate group Euglenozoa.

\subsection{A Potential Secondary RNP-4F Chaperone Recognition Site in the 5'-UTR of Drosophila rnp-4f Pre-mRNA May Play a Key Role in Controlling Its Own Expression}

We have previously described a long evolutionarilyconserved potential stem-loop which arises by basepairing between all of the $r n p-4 f$ pre-mRNA intron 0 and part of adjacent exon 2 in $D$. melanogaster $[6,8]$. We have recently shown using RNA electrophoretic mobility shift assay that retention of intron 0 within the $r n p-4 f$ 5'-UTR is correlated with binding of a dADAR protein isoform, and that an unidentified second protein suspected to be RNP-4F also binds to this stem-loop [9]. Subsequent work employing RNAi technology showed that this dADAR protein is the truncated isoform [11]. We have proposed a negative feedback model for regulating expression of $r n p-4 f$ mRNA under conditions of RNP-4F excess within the developing fly central nervous system [6]. If this hypothesis is correct, then the conserved long stem-loop would be expected to contain a nucleotide recognition sequence to which RNP-4F could potentially bind, in competition with its preferred binding to a conserved sequence tract within the long stemloop of free U6-snRNA [13]. In Drosophila U6-snRNA the conserved sequence contains nucleotides between positions \#38-57, although an even shorter sequence may suffice for chaperone binding, but this possibility has not yet been tested. Examination of the Drosophila conserved $r n p-4 f$ 177-nt stem-loop nucleotide sequence/ structure shows that a 12-nt tract closely resembling the preferred U6-snRNA binding site is indeed present (Figure 5(b), (c)). An additional similarity between the 


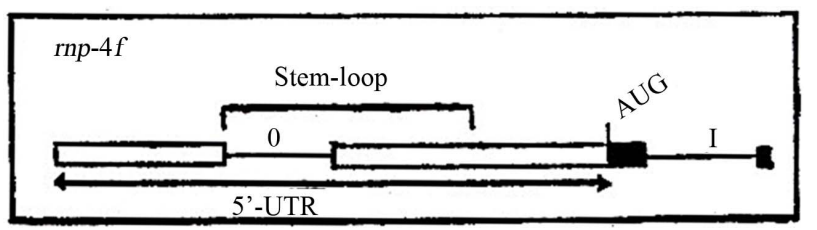

(a)

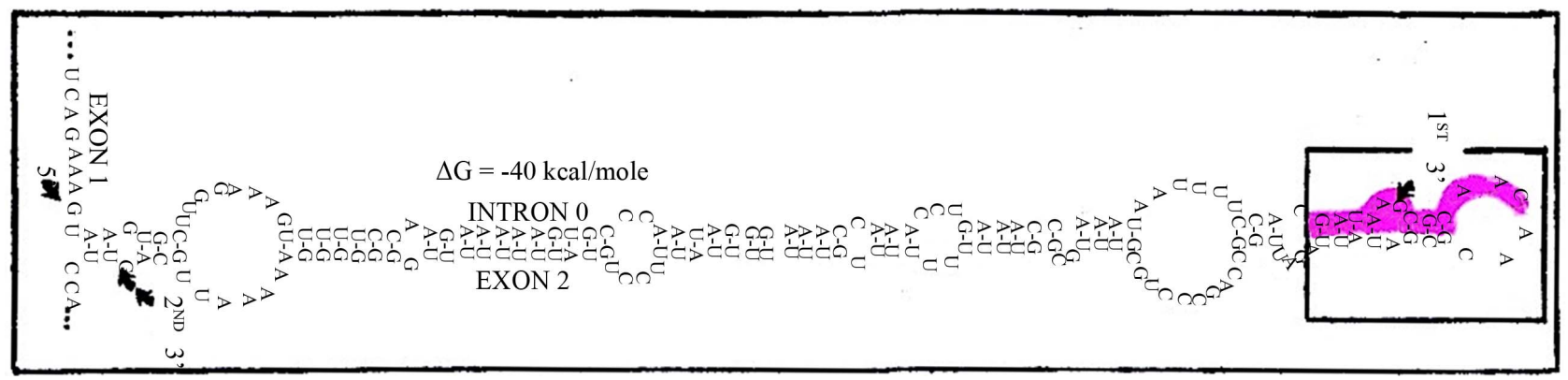

(b)

\begin{tabular}{|c|c|c|}
\hline U6-snRNA & D. melanogaster & $\mathrm{G} A \mathrm{~A} U \mathrm{U}$ A $\mathrm{G}$ C A U G $\mathrm{G}$ C \\
\hline U6-snRNA & H. sapiens & $\mathrm{G} A \mathrm{~A} \quad \mathrm{U}$ A $\mathrm{G} \quad \mathrm{C} A \mathrm{~A} \quad \mathrm{U} \quad \mathrm{G} \quad \mathrm{G} \quad \mathrm{C}$ \\
\hline U6-snRNA & S. pombe & 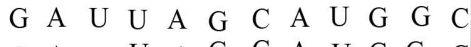 \\
\hline U6-snRNA & A. thaliana & 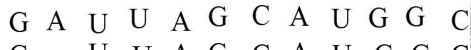 \\
\hline \multirow[t]{3}{*}{ U6-snRNA } & T. thermophila & $G$ A $U$ U A $A$ C A $A$ G $G$ C \\
\hline & rnp- $4 f$ pre-mRNA & 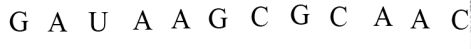 \\
\hline & CONSENSUS & 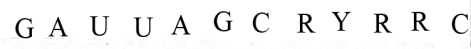 \\
\hline
\end{tabular}

(c)

Figure 5. A 177-nt long Drosophila rnp-4f stem-loop in the pre-mRNA 5'-UTR regulatory region contains a potential RNP-4F protein chaperone binding site. (a) Orientation diagram showing position of long stem-loop which forms by hydrogen bonding between intron 0 and part of exon 2. (b) Long interrupted rnp-4f stem-loop secondary structure as predicted from Mfold program (29). The 5'and 3'-limits of intron 0 are indicated, in addition to alternative 3'-splice site within exon 2 (8) and evolutionarily-conserved short stem-loop (boxed) at tip of the longer structure (6). The highlighted nucleotides near the tip show position of potential RNP-4F protein binding site postulated to compete with the preferred experimentally determined tract within U6-snRNA (13). (c) Alignment at region of chaperone RNP-4F/Prp24/p110 binding site to U6-snRNA in various species, and to potential rnp-4f pre-mRNA nucleotides.

RNP-4F chaperone substrate free U6-snRNA (Figure 4(a)) and $r n p-4 f$ pre-mRNA is that in both cases the recognition sequence is contained within a long, interrupted stem-loop structure. In Drosophila free U6- snRNA this stem-loop contains 81-nt, while in rnp-4f the stem-loop contains 177-nt. Finally, RNP-4F is a nuclear protein (6) and thus would be expected to have access to the long stem-loop in rnp-4f pre-mRNA. These observations support the hypothesis that excess RNP-4F protein may competitively bind to a 5'-UTR regulatory region within its own pre-mRNA, playing a role in negative feedback control.

\section{CONCLUSION}

Our long standing interest in Drosophila splicing assembly factor RNP-4F, which functions as a chaperone to facilitate bonding between U4- and U6-snRNA, led us to analyze the secondary structure of the U4-/U6-snRNA duplex. Close study of published chemical and enzymatic probing results on the proposed human and yeast $S$. cerevisiae U4-/U6-snRNA structures [37] suggested to us certain inconsistencies within the classical Y-shaped model [12]. Further, preliminary comparison of the classical model with a computer-generated secondary structure also revealed inconsistencies, which led us to reexamine this model. We deemed this timely in light of the many new U4-and U6-snRNA sequences that have become available, in large part, by recent genomic sequencing projects. Our study, utilizing the comparative phylogenetic approach, eventually resulted in a revised and improved U4-/U6-snRNA secondary structure model. The model proven by observation of abundant compensatory base mutations in every stem is shown to be general but not universal, and structural variations have been traced to their origins within the phylogenetic tree. We have extensively probed the eukaryotic tree to its deepest roots, and our results suggest that U4- and U6- 
snRNAs apparently evolved after the emergence of lines leading to the diplomonad Giardia and the parabasalid Trichomonas, but once established they have maintained a remarkably well conserved U4-/U6-snRNA secondary structure extending to, and including, the flagellates among the Euglenozoa. An unexpected result of this study was discovery of a potential competitive binding site for Drosophila splicing assembly factor RNP-4F to a 5'-UTR regulatory region within its own pre-mRNA, which may play a role in negative feedback control [6]. This negative feedback expression control model awaits experimental testing.

\section{ACKNOWLEDGEMENTS}

We wish to thank the late Carl Woese for his helpful discussions of RNA secondary structure determinations over more than two decades, to whom this study is dedicated. We also thank Jane Carlton and Steven Sullivan for helpful discussion bearing on Giardia and Trichomonas splicing machinery components. Peter Dobler is to be thanked for his help in constructing the secondary structure figures in the manuscript. This work was primarily supported by National Institutes of Health (NIH) Grant 1-R15-GM070802-01 and 1-R15-GM093895-01 to J. Vaughn.

\section{REFERENCES}

[1] Banfi, S., Borsani, G., Rossi, E., Bernard, L., Guffanti, A., Rubboli, F., Marchitiello, A., Giglio, S., Coluccia, E. and Zollo, M. (1996). Identification and mapping of human cDNAs homologous to Drosophila mutant genes through EST database searching. Nature Genetics, 13, 167-174. http://dx.doi.org/10.1038/ng0696-167

[2] Fortini, M.E., Skupski, M.P., Boguski, M.S. and Hariharan, I.K. (2000) A survey of human disease gene counterparts in the Drosophila genome. The Journal of Cell Biology, 150, F23-F30.

http://dx.doi.org/10.1083/jcb.150.2.F23

[3] Petschek, J.P., Scheckelhoff, M.R., Mermer, M.J. and Vaughn, J.C. (1997) RNA editing and alternative splicing generate mRNA transcript diversity from the Drosophila 4f-rnp locus. Gene, 204, 267-276.

http://dx.doi.org/10.1016/S0378-1119(97)00465-4

[4] Feiber, A.L., Rangarajan, J. and Vaughn, J.C. (2002) The evolution of single-copy Drosophila nuclear 4f-rnp genes, spliceosomal intron losses create polymorphic alleles. Journal of Molecular Evolution, 55, 401-413. http://dx.doi.org/10.1007/s00239-002-2336-y

[5] Peters, N.T., Rohrbach, J.A., Zalewski, B.A., Byrkett, C.M. and Vaughn, J.C. (2003) RNA editing and regulation of Drosophila $4 f$-rnp expression by sas-10 antisense readthrough mRNA transcripts. $R N A, \mathbf{9}, 698-710$. http://dx.doi.org/10.1261/rna.2120703

[6] Fetherson, R.A., Strock, S.B., White, K.N. and Vaughn, J.C. (2006) Alternative pre-mRNA splicing in Drosophila spliceosomal assembly factor RNP-4F during development. Gene, 371, 234-245.

\section{http://dx.doi.org/10.1016/j.gene.2005.12.025}

[7] Chen, J., Concel, V.J., Bhatla, S., Rajeshwaran, R., Smith, D.L.H., Varadarajan, M., Backscheider, K.L., Bockrath, R.A., Petschek, J.P. and Vaughn, J.C. (2007) Alternative splicing of an $r n p-4 f$ mRNA isoform retaining an evolutionarily-conserved 5'-UTR intronic element is developmentally regulated and shown via RNAi to be essential for normal central nervous system development in Drosophila melanogaster. Gene, 399, 91-104.

http://dx.doi.org/10.1016/j.gene.2007.04.038

[8] Chen, J., Lakshmi, G.G., Hays, D.L., McDowell, K.M., Ma, E. and Vaughn, J.C. (2009) Spatial and temporal expression of $d A D A R$ mRNA and protein isoforms during embryogenesis in Drosophila melanogaster. Differentiation, 78, 312-320.

http://dx.doi.org/10.1016/j.diff.2009.08.003

[9] Lakshmi, G.G., Ghosh, S., Jones, G.P., Parikh, R., Rawlins, B.A. and Vaughn, J.C. (2012) An RNA electrophoretic mobility shift and mutational analysis of $r n p-4 f$ 5'-UTR intron splicing regulatory proteins in Drosophila reveals a novel new role for a dADAR protein isoform. Gene, 511, 161-168. http://dx.doi.org/10.1016/j.gene.2012.09.088

[10] Chen, J., Yang, J.T., Doctor, D.L., Rawlins, B.A., Shields, B.C. and Vaughn, J.C. (2013) 5'-UTR mediated translational control of splicing assembly factor RNP-4F expression during development of the Drosophila central nervous system. Gene, 528,154-162.

[11] Ghosh, S., Wang, Y., Cook, J.A., Chhiba, L. and Vaughn, J.C. (2013) A molecular, phylogenetic and functional study of the $d A D A R$ mRNA truncated isoform during Drosophila embryonic development. Open Journal of Animal Sciences, (In press).

[12] Brow, D.A. and Guthrie, C. (1988) Spliceosomal RNA U6 is remarkably conserved from yeast to mammals. $\mathrm{Na}$ ture, 334, 213-218. http://dx.doi.org/10.1038/334213a0

[13] Bell, M., Schreiner, S., Damianov, A., Reddy, R. and Bindereif, A. (2002) p110, a novel human U6 snRNP protein and U4/U6 snRNP recycling factor. The $E M B O$ Journal, 21, 2724-2735. http://dx.doi.org/10.1093/emboj/21.11.2724

[14] Rader, S.D. and Guthrie, C. (2002) A conserved Lsminteraction motif in Prp24 required for efficient U4/U6 di-snRNP formation. RNA, 8, 1378-1392. http://dx.doi.org/10.1017/S1355838202020010

[15] Karaduman, R., Fabrizio, P., Hartmuth, K., Urlaub, H. and Luhrmann, R. (2006) RNA structure and RNA-protein interactions in purified yeast U6 snRNPs. Journal of Molecular Biology, 356, 1248-1262.

http://dx.doi.org/10.1016/j.jmb.2005.12.013

[16] Bae, E., Reiter, N.J., Bingman, C.A., Kwan, S.S., Lee, D., Phillips, G.N., Butcher, S.E. and Brow, D.A. (2007) Structure and interactions of the first three RNA recognition motifs of splicing factor Prp24. Journal of Molecular Biology, 367, 1447-1458. http://dx.doi.org/10.1016/j.jmb.2007.01.078

[17] Moore, M.J., Query, C.C. and Sharp, P.A. (1993) Splicing of precursors to mRNA by the spliceosome. In: Gesteland, R.F. and Atkins, J.F., Ed., The RNA World. Cold Spring Harbor Laboratory Press, Cold Spring Harbor, New York, 303-357. 
[18] Will, C.L. and Luhrmann, R. (2006) Spliceosome structure and function. In: Gesteland, R.F., Cech, T.R. and Atkins, J.F., Ed., The RNA World. Cold Spring Harbor Laboratory Press, Cold Spring Harbor, New York, 369-400.

[19] Jurica, M.S. and Moore, M.J. (2003) Pre-mRNA splicing, awash in a sea of proteins. Molecular Cell, 12, 5-14. http://dx.doi.org/10.1016/S1097-2765(03)00270-3

[20] Noller, H.F., Kop, J.A., Wheaton, V., Brosius, J., Gutell, R.R., Kopylov, A.M., Dohme, F., Herr, W., Stahl, D.A., Gupta, R. and Woese, C.R. (1981) Secondary structure model for 23S ribosomal RNA. Nucleic Acids Research, 9, 6167-6189. http://dx.doi.org/10.1093/nar/9.22.6167

[21] Vaughn, J.C., Sperbeck, S.J., Ramsey, W.J. and Lawrence, C.B. (1984) A universal model for the secondary structure of 5.8S ribosomal RNA molecules, their contact sites with $28 \mathrm{~S}$ ribosomal RNAs, and their prokaryotic equivalent. Nucleic Acids Research, 12, 7479-7502. http://dx.doi.org/10.1093/nar/12.19.7479

[22] Orum, H., Nielsen, H. and Engberg, J. (1991) Spliceosomal small nuclear RNAs of Tetrahymena thermophila and some possible snRNA-snRNA base-pairing interactions. Journal of Molecular Biology, 222, 219-232. http://dx.doi.org/10.1016/0022-2836(91)90208-N

[23] Hofmann, C.J.B., Marshallsay, C., Waibel, F. and Filipowicz, W. (1992) Characterization of the genes encoding U4 small nuclear RNAs in Arabidopsis thaliana. Molecular Biology Reports, 17, 21-28. http://dx.doi.org/10.1007/BF01006396

[24] Jakab, G., Mougin, A., Kis, M., Pollak, T., Antal, M., Branlant, C. and Solymosy, F. (1997) Chlamydomonas U2, U4 and U6 snRNAs. An evolutionary conserved putative third interaction between $\mathrm{U} 4$ and U6 snRNAs which has a counterpart in the $\mathrm{U} 4_{\text {atac }}-\mathrm{U} 6_{\text {atac }}$ snRNA duplex. Biochimie, 79, 387-395. http://dx.doi.org/10.1016/S0300-9084(97)86148-2

[25] Hinas, A., Larsson, P., Avesson, L., Kirsebom, L.A., Virtanen, A. and Soderbom, F. (2006) Identification of the major spliceosomal RNAs in Dictyostelium discoideum reveals developmentally regulated U2 variants and polyadenylated snRNAs. Eukaryot. Cell, 5, 924-934. http://dx.doi.org/10.1128/EC.00065-06

[26] Davis, C.A., Brown, M.P.S. and Singh, U. (2007) Functional characterization of spliceosomal introns and identification of U2, U4, and U5 snRNAs in the deep-branching eukaryote Entamoeba histolytica. Eukaryotic Cell, 6, 940-948. http://dx.doi.org/10.1128/EC.00059-07

[27] Gu, J., Chen, Y. and Reddy, R. (1998) Small RNA database. Nucl. Acids Res. 26, 160-162. http://dx.doi.org/10.1093/nar/26.1.160

[28] Stark, A., Lin, M.F., Kheradpour, P., et al. (2007) Discovery of functional elements in 12 Drosophila genomes using evolutionary signatures. Nature, 450, 219-232. http://dx.doi.org/10.1038/nature06340

[29] Zuker, M. (2003) Mfold web server for nucleic acid folding and hybridization prediction. Nucleic Acids Research, 31, 3406-3415. http://dx.doi.org/10.1093/nar/gkg595

[30] Rinke, J., Appel, B., Digweed, M. and Luhrmann, R. (1985) Localization of a base paired interaction between small nuclear RNAs U4 and U6 in intact U4/U6 ribonucleoprotein particles by psoralem cross-linking. Journal of Molecular Biology, 185, 721-731. http://dx.doi.org/10.1016/0022-2836(85)90057-9

[31] Steenkamp, E.T., Wright, J. and Baldauf, S.L. (2006). The protistan origins of animals and fungi. Molecular Biology and Evolution, 23, 93-106. http://dx.doi.org/10.1093/molbev/msj011

[32] Myslinski, E. and Branlant, C. (1991) A phylogenetic study of U4 snRNA reveals the existence of an evolutionarily conserved secondary structure corresponding to "free" U4 snRNA. Biochimie, 73, 17-28. http://dx.doi.org/10.1016/0300-9084(91)90069-D

[33] Mitrovich, Q.M. and Guthrie, C. (2007) Evolution of small nuclear RNAs in S. cerevisiae, C. albicans, and other hemiascomycetous yeasts. RNA, 13, 2066-2080. http://dx.doi.org/10.1261/rna.766607

[34] Krol, A., Branlant, C., Lazar, E., Gallinaro, H. and Jacob, M. (1988) Primary and secondary structures of chicken, rat and man nuclear U4 RNAs. Homologies with U1 and U5 RNAs. Nucleic Acids Research, 9, 2699-2716. http://dx.doi.org/10.1093/nar/9.12.2699

[35] Epstein, P., Reddy, R., Henning, D. and Busch, H. (1980). The nucleotide sequence of nuclear U6 (4.7S) RNA. Journal of Molecular Biology, 255, 8901-8906.

[36] Fortner, D.M., Troy, R.G. and Brow, D.A. (1994) A stem/loop in U6 RNA defines a conformational switch required for pre-mRNA splicing. Genes \& Development, 8, 221-233. http://dx.doi.org/10.1101/gad.8.2.221

[37] Mougin, A., Gottschalk, A., Fabrizio, P., Luhrmann, R. and Branlant, C. (2002) Direct probing of RNA structure and RNA-protein interactions in purified HeLa cell's and yeast spliceosomal U4/U6.U5 tri-snRNP particles. Journal of Molecular Biology, 317, 631-649. http://dx.doi.org/10.1006/jmbi.2002.5451

[38] Stark, H. and Luhrmann, R. (2006) Cryo-electron microscopy of spliceosomal components. Annual Review of Biophysics and Biomolecular Structure, 35, 435-457. http://dx.doi.org/10.1146/annurev.biophys.35.040405.101 $\underline{953}$

[39] McArthur, A.G., Morrison, H.G., Nixon, J.E.J., et al. (2000) The Giardia genome project database. FEMS Microbiology Letters, 189, 271-273. http://dx.doi.org/10.1111/j.1574-6968.2000.tb09242.x

[40] Carlton, J.M., Hirt, R.P., Silva, J.C., et al. (2007) Draft genome sequence of the sexually transmitted pathogen Trichomonas vaginalis. Science, 315, 207-212. http://dx.doi.org/10.1126/science.1132894

[41] Bonen, L. and Vogel, J. (2001) The ins and outs of group II introns. Trends in Genetics, 17, 322-331. http://dx.doi.org/10.1016/S0168-9525(01)02324-1

[42] Fedorova, O. and Zingler, N. (2007) Group II introns, structure, folding and splicing mechanism. The Journal of Biological Chemistry, 388, 665-678. http://dx.doi.org/10.1515/BC.2007.090

[43] Vanacova, S., Yan, W., Carlton, J.M. and Johnson, P.J. (2005) Spliceosomal introns in the deep-branching eukaryote Trichomonas vaginalis. Proceedings of the $\mathrm{Na}$ tional Academy of Sciences, 102, 4430-4435. http://dx.doi.org/10.1073/pnas.0407500102 